\title{
Pharmaceutical Informatics and the Pathway to Personalized Medicines
}

\author{
Sangtae Kim* and Venkat Venkatasubramanian \\ Purdue University, West Lafayette, IN 47907, USA \\ kim55@purdue.edu
}

\begin{abstract}
The present computational era has witnessed the evolution of the field of computational biology into two somewhat disjoint communities: one group focused on the "wriggling of molecules" (e.g. molecular dynamics) and another focused on pattern matching and informatics (e.g. sequence alignment). The separation starts relatively early in the training of the community, as is evident from the relative weighting of calculus vs. computer science courses in the curriculum of undergraduate majors in the various disciplines of computational biology. But at the dawn of the petascale era in computational science, exciting advances in healthcare can be envisioned at a new niche in the computational biology ecosystem - a niche formed by the reunion of the separated branches of computational biology. We consider some illustrative examples from pharmaceutical informatics of data-intensive informatics approaches applied to "objects" that are not simply numbers or static molecular structures but instead are (CPU-intensive) molecular simulations. These results suggest a promising pathway for applying petascale informatics to shift the paradigm in pharmaceutical and biotechnology $R \& D$ to a future world of personalized medicines. The early examples from pharmaceutical informatics also indicate that the foundational framework will be based on the concept of Dynamic Data Driven Application Systems (DDDAS).
\end{abstract}

* Conference speaker. 